\title{
MOSQUITO TRYPANOSOMES.
}

\author{
By F. G. NOVY, W. J. MACNEAL AND H. N. TORREY \\ (Ann Arbor, Michigan).
}

IN a previous paper on Bird Trypanosomes it was pointed out that these organisms grew readily in the test-tube on blood agar and that the resulting forms resembled the flagellates which Schaudinn found in the gut of mosquitoes which had fed on owls infected with Halteridium and with H. Ziemanni. In other words, the position taken was that the flagellates observed in the mosquitoes did not represent stages in the life-history of intracellular parasites but were actually cultures in vivo of trypanosomes present in the blood of the birds used. In confirmation of this position it was desirable to show that trypanosomes could actually grow and multiply in the gut of mosquitoes and that such forms actually did correspond to those which would be obtained in vitro.

Accordingly, large numbers of mosquitoes were captured along the river-bank and allowed to feed on perfectly clean animals, such as rats, guinea-pigs and pigeons. At varying intervals, 36 to 72 hours after feeding, the contents of the stomachs of the mosquitoes were examined in living and in stained preparations and cultures on blood agar were made at the same time. Of more than 800 mosquitoes which were examined in this way about 120 or 15 per cent. were found to have a flagellate infection of the intestinal tract. In some, this was very marked; large masses of rosettes, flagella inside, completely filling the lumen of the tube.

Several distinct forms of the trypanosomes were met with, the most common of these was a Herpetomonas (probably Herpetomonas subulata) and Chrithidia fasciculata.

Owing to the large numbers of bacteria usually present much difficulty was experienced in obtaining cultures of these flagellates. Eventually, however, the Herpetomonas was isolated in mixed culture associated with a minute coccus, while the Chrithidia was obtained in association with a yeast. These mixed cultures have now been grown in the laboratory for some six months. Several other cultures were obtained but these were soon outgrown by the accompanying bacteria.

The cultural form of these two organisms was exactly the same as that seen in the gut of the mosquito, thus confirming the view expressed that the flagellates found growing in the intestinal tube of insects represent cultural forms in vivo, and, as such, correspond to those in vitro. In both conditions not only was the form and size the same but the blepharoplast was anterior to the nucleus. The Herpetomonas was characterized by the presence of two diplosomes in the posterior part of the cell. These bodies were found in the parasites within the mosquitoes as well as those grown in culture. Animals inoculated with the cultures failed to show an infection.

When mosquitoes are allowed to feed on Tr. Brucei or Tr. Lewisi these parasites may be detected in the blood in the intestine of the mosquito 24 hours after feeding, and even later, and rats inoculated with such stomach contents develop typical infection. 